\title{
Effect of the local application of bupivacaine in early pain control following impacted mandibular third molar surgery: A randomized controlled study
}

\author{
Mohanad Ali Shabat ${ }^{B, D, F}$, Salwan Yousif Bede ${ }^{A, C, E}$ \\ Department of Oral and Maxillofacial Surgery, College of Dentistry, University of Baghdad, Baghdad Medical City, Bab-Almoadham, Baghdad, Iraq \\ A - research concept and design; $\mathrm{B}$ - collection and/or assembly of data; $\mathrm{C}$ - data analysis and interpretation; \\ $D$ - writing the article; $E$ - critical revision of the article; $F$ - final approval of the article
}

Address for correspondence

Mohanad Ali Shabat

E-mail:mohanadshabat@gmail.com

Funding sources

None declared

Conflict of interest

None declared

Received on December 12, 2020

Reviewed on February 20, 2021

Accepted on February 24, 2021

Published online on December 31, 2021

Cite as

Shabat MA, Bede SY. Effect of the local application of bupiva-

caine in early pain control following impacted mandibular third molar surgery: A randomized controlled study. Dent Med Probl. 2021;58(4):483-488. doi:10.17219/dmp/133664

DOI

$10.17219 / \mathrm{dmp} / 133664$

Copyright

๑ 2021 by Wroclaw Medical University

This is an article distributed under the terms of the

Creative Commons Attribution 3.0 Unported License (CC BY 3.0)

(https://creativecommons.org/licenses/by/3.0/).

\begin{abstract}
Background. Postoperative pain is one of the main complications following impacted mandibular third molar (IMTM) surgery.

Objectives. The aim of this study was to assess the effect of the local application of bupivacaine on reducing early postoperative pain following IMTM surgery.

Material and methods. A prospective, single-blinded, randomized controlled study was conducted on 40 patients who had undergone the surgical removal of an IMTM under local anesthesia. In the study group ( $n=20)$, absorbable gelatin sponge (AGS) soaked in $3 \mathrm{~mL}$ of $0.5 \%$ plain bupivacaine hydrochloride was locally applied in the post-extraction socket. In the control group $(n=20)$, AGS soaked in $3 \mathrm{~mL}$ of normal saline was used. Pain intensity was assessed using a pain numerical rating scale (NRS) 4 and $12 \mathrm{~h}$ postoperatively. The variables were compared between the 2 groups and probability values $<0.05$ were considered statistically significant.

Results. The pain scores in the study group were significantly lower than those recorded in the control group at $4 \mathrm{~h}$ postoperatively $(p=0.003)$, whereas the difference in the pain scores between the 2 groups $12 \mathrm{~h}$ after surgery was not statistically significant $(p=0.443)$.

Conclusions. The local application of bupivacaine is effective in reducing postoperative pain $4 \mathrm{~h}$ after the surgical extraction of IMTMs without any significant complications.
\end{abstract}

Keywords: third, postoperative, pain, bupivacaine, molar 


\section{Introduction}

The impaction of third molars is a common problem worldwide. Its prevalence has been estimated to range from about $3 \%$ to about $68 \%$, with an average rate of $24.4 \%$; it has significant geographic variation. ${ }^{1}$ The surgical extraction of impacted mandibular third molars (IMTMs) is a common practice in oral surgery and it is usually accompanied by side effects, such as swelling, trismus and postoperative pain. ${ }^{2-5}$

Postoperative pain is the most common complication following dental surgery, ${ }^{3}$ and the patient's experience depends on the degree of surgical trauma, the need for bone removal and the periosteal extension. It is undoubtedly the most common symptom and can continue even 1 week after surgery, despite analgesic treatment, leading to increased morbidity and detrimental effects on the patient's well-being and quality of life. ${ }^{6,7}$ Inadequate management of early postoperative pain has been related to a wide range of negative outcomes, including delayed recovery and even an increased risk of developing chronic pain. ${ }^{8,9}$

The development and use of local anesthetics for intraoperative anesthesia and postoperative analgesia is considered to be one of the most important advances in dentistry in the last century. ${ }^{10}$ Several researchers have credited bupivacaine with the ability to provide prolonged postoperative analgesia and to minimize analgesic requirements in the early postoperative hours, when pain is at its highest intensity. Its long duration of action and superior ability to decrease pain and discomfort have been reported in comparison with articaine and lidocaine. Therefore, bupivacaine is a common choice for pain control following IMTM extraction. ${ }^{11}$

The delivery of a local anesthetic agent to the postextraction socket has been described to alleviate postoperative pain. ${ }^{3}$ However, the use of local delivery therapeutics requires some mechanism to maintain the agent within the oral environment for an adequate period of time to prolong drug effectiveness and reduce its toxicity by slowing drug uptake into the systemic circulation. ${ }^{12,13}$

Few studies have investigated the effect of the local application of bupivacaine in post-extraction sockets following the surgical removal of IMTMs. Therefore, the aim of this study was to assess the effect of the local application of bupivacaine on reducing early postoperative pain following IMTM surgery.

\section{Material and methods}

This study was designed and conducted as a prospective, single-blinded, randomized controlled study guided by the Consolidated Standards of Reporting Trials (CONSORT) statement. ${ }^{14}$ It included patients undergoing the surgical extraction of IMTMs at the Department of Oral and Maxillofacial Surgery of the College of Dentistry, University of Baghdad, Iraq.
The inclusion criteria were as follows: adults aged 18 years or older; with the American Society of Anesthesiologists physical status classification level I (ASA I); who had an IMTM indicated for surgical extraction; and reported no history of allergic reactions to the local anesthetic agents used in the study.

The exclusion criteria embraced patients with systemic diseases, pregnant females, patients with a missing lower second molar on the side of the surgery, patients with impacted teeth due to any pathology, such as cysts or tumors, patients presenting with signs and symptoms of acute general infections, such as fever and lymphadenopathy, and patients who had taken any type of analgesic within $24 \mathrm{~h}$ prior to the surgery.

The study was approved by the institutional Research Ethics Committee (protocol No. 127119). The patients were informed about the nature of the study, the procedures, and the possible intra- and postoperative complications they might encounter. Every patient signed an informed consent form to participate in the study.

The patients were randomly assigned to 2 groups with the use of Microsoft Office Excel 2016 (Microsoft Corporation, Redmond, USA). Block randomization was performed to ensure a nearly equal distribution of patients in both groups. For the patients in the study group, a $10 \times 10 \times 10-\mathrm{mm}$ piece of absorbable gelatin sponge (AGS) (SPONGOSTAN ${ }^{\circledR}$ Dental; Ferrosan Medical Devices, Søborg, Denmark) soaked in $0.5 \%$ plain bupivacaine hydrochloride (Marcaine; AstraZeneca, Istanbul, Turkey) was applied in the post-extraction socket. The patients assigned to the control group received AGS soaked in normal saline as a placebo. The patients were blinded to the type of local treatment they received.

A preoperative panoramic radiograph was obtained for each patient to assess the angulation of the impacted tooth according to Winter's classification ${ }^{15}$ as well as the position and depth of impaction according to the Pell and Gregory classification. ${ }^{16}$ All procedures were scheduled to start at 9 a.m. and were performed by one operator under local anesthesia ( $2 \%$ lidocaine hydrochloride with epinephrine 1:80,000). The procedure consisted of reflecting a triangular flap and extracting the tooth with the use of elevators alone, or after bone removal with or without tooth sectioning with the use of a surgical handpiece and burs under copious irrigation with normal saline. The duration of the surgery was calculated in minutes starting from the first incision to the last suture.

Operative difficulty was determined by the surgical technique and the duration of extraction according to de Carvalho and Vasconcelos. ${ }^{17}$ With respect to the surgical technique, the degree of difficulty was considered low when the extraction was performed using elevators alone, moderate when bone removal was required, and high when bone removal and tooth sectioning were required to complete the extraction. For the duration of surgery, the difficulty was considered low when the duration 
of the surgical extraction was less than 15 min, moderate when the duration was 15-30 min and high when the duration was more than $30 \mathrm{~min}$.

After extraction, the socket was thoroughly irrigated with normal saline, all sharp edges were smoothened and hemostasis was achieved. In the study group, a piece of AGS was soaked in $3 \mathrm{~mL}$ of $0.5 \%$ plain bupivacaine hydrochloride for $5 \mathrm{~min}$ and applied in the post-extraction socket. In the control group, the same procedure was performed, but $3 \mathrm{~mL}$ of normal saline was used. The flap was repositioned to its original place and sutured with $3 / 0$ black silk sutures, using the simple interrupted suturing technique. Antibiotics (amoxicillin $500 \mathrm{mg}$ every $8 \mathrm{~h}$ for 5 days) and ibuprofen ( $400 \mathrm{mg}$ every $8 \mathrm{~h}$ for 5 days) were prescribed to all patients, and the patients were instructed to record any extra analgesic drug use.

Pain intensity was reported by the patients on a pain numerical rating scale (NRS) 4 and $12 \mathrm{~h}$ postoperatively. The patients were instructed to write a number $(0-10)$ to quantify their pain intensity, where 0 represented no pain and 10 represented the worst pain possible. The patients were aware that the scale served to analyze the presence and intensity of pain alone, and was not a representation of generalized postoperative discomfort.

The predictor variable was the local application of bupivacaine- or normal saline-soaked AGS in the postextraction socket, while the outcome variable was the pain scores recorded 4 and $12 \mathrm{~h}$ after surgery. Other variables included age, gender, indication for extraction, the angulation, position and depth of the IMTM, and the difficulty of extraction determined by the surgical technique and the duration of extraction.

\section{Statistical analysis}

The statistical analysis was performed using GraphPad Prism v. 6 for Windows (GraphPad Software, La Jolla, USA). The descriptive statistical analysis included the calculation of frequency as number $(n)$, mean $(M)$ and standard deviation $(S D)$, and median $(M e)$. The inferential analysis included the Shapiro-Wilk normality test, the Mann-Whitney $U$ test, Cohen's $d$ test, Fisher's exact test, and the $X^{2}$ test. Probability values $<0.05$ were considered statistically significant.

\section{Results}

Forty-three patients initially participated in this study, but 3 patients were lost to follow-up and excluded from the analysis. The remaining 40 patients ranged in age from 18 to 30 years, with a mean age of $23.6 \pm 3.55$ years. Among the patients there were 17 (42.5\%) males and $23(57.5 \%)$ females. They were randomly assigned to the control and study groups of 20 patients each. Figure 1 summarizes the phases of the study.

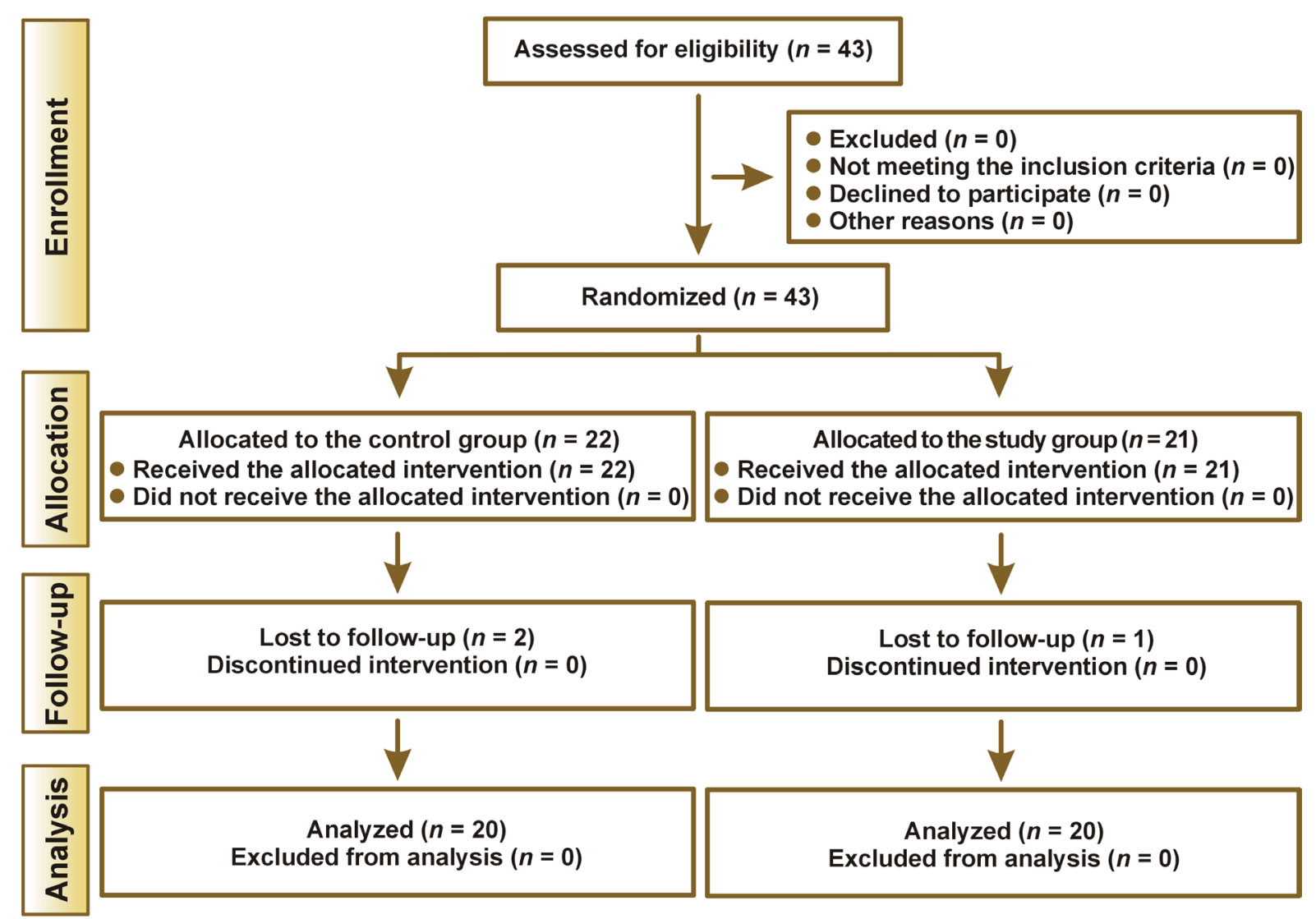

Fig. 1. Flow diagram of the randomized study 
The comparisons of different independent variables between the 2 groups are summarized in Table 1. All differences were statistically non-significant, which indicates that none of these variables acted as a confounding factor for the outcome of interest (the pain score).

Four hours postoperatively, the study group recorded a mean pain score of $2.90 \pm 2.43$ and a median of 3.0, while the control group recorded a mean pain score of $5.95 \pm 3.14$ and a median of 5.5. The difference in the pain scores between the 2 groups was statistically significant $(p=0.003)$ (Fig. 2). The effect size of the intervention was 0.9723 (Glass's delta), which can be interpreted as a large effect (large effect: 0.8 or more).

Twelve hours after surgery, the pain scores recorded by the study group presented a mean of $3.80 \pm 3.09$ and a median of 3.0, while the control group had a mean of $4.25 \pm 2.71$ and a median of 3.0. The difference between the 2 groups for this time interval was not statistically significant $(p=0.443)$ (Fig. 2).

None of the patients in either group required extra analgesics other than those prescribed during the first $24 \mathrm{~h}$ postoperatively. The sutures were removed 7 days postoperatively. Five patients in the control group developed

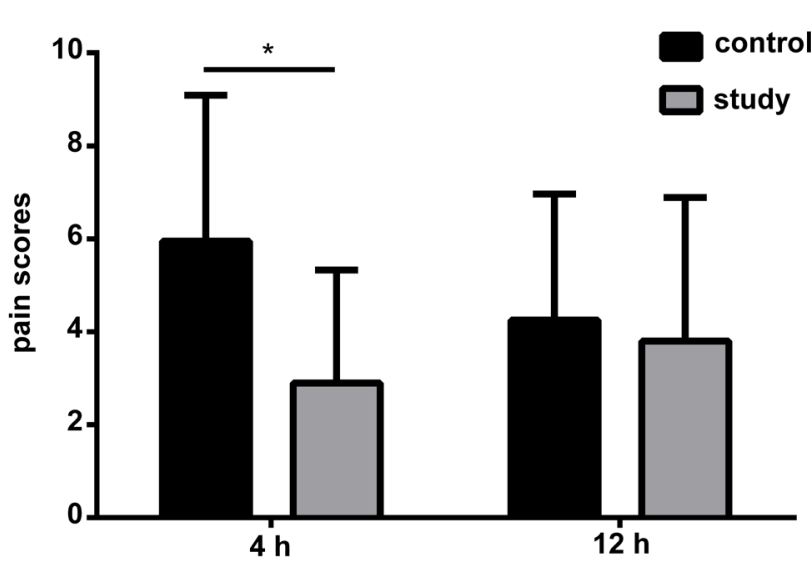

Fig. 2. Bar chart illustrating the difference in the pain scores between the 2 groups 4 and $12 \mathrm{~h}$ postoperatively

* statistically significant.

complications (a dry socket in 2 patients and wound dehiscence in 3 patients). Three patients in the study group developed complications (a dry socket in 2 patients and wound dehiscence in 1 patient). The difference in complication rates between the 2 groups was not statistically significant $(p=0.695)$.

Table 1. Comparison of independent variables between the 2 groups

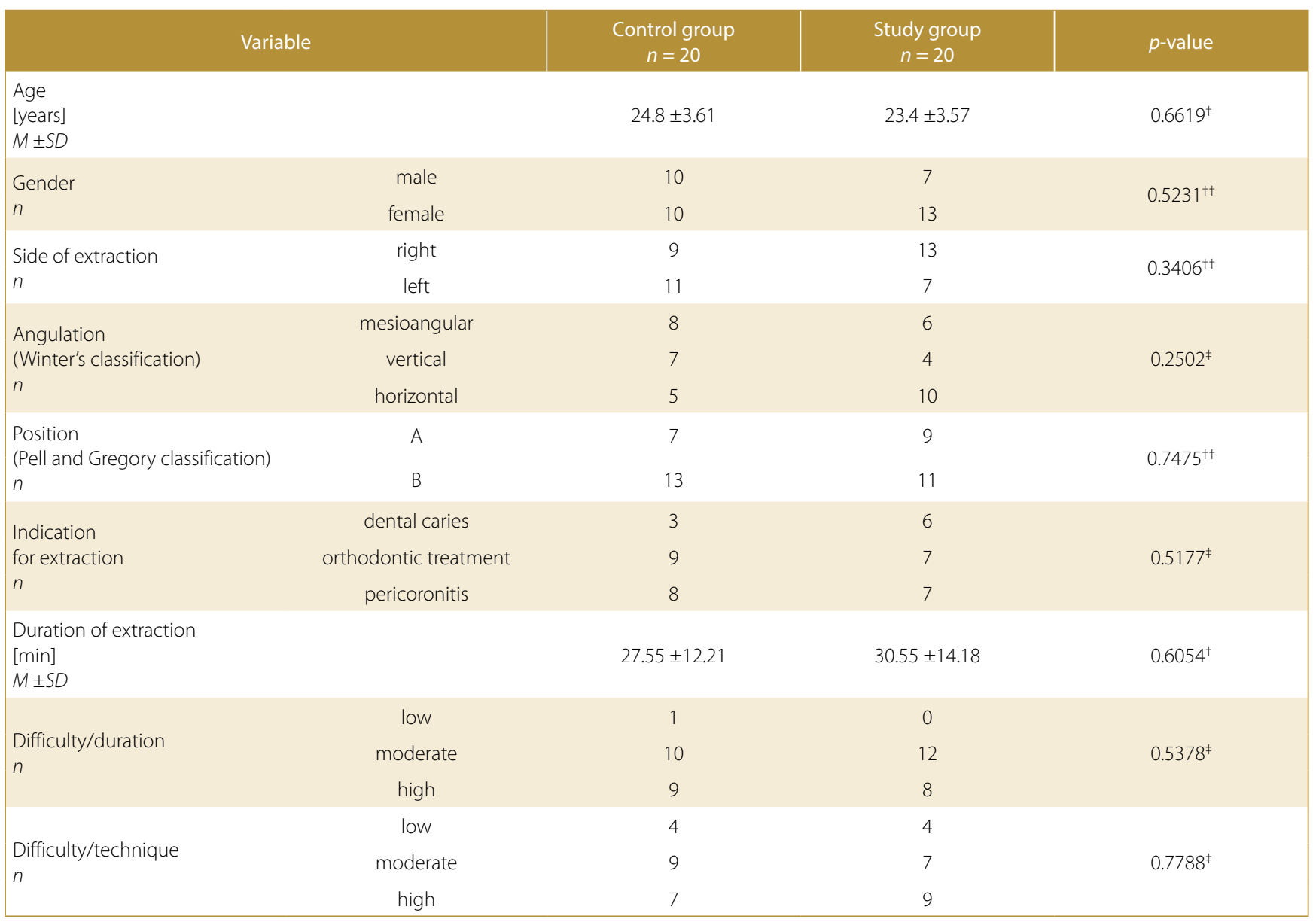

$n$ - number; $M$ - mean; SD - standard deviation; ${ }^{\dagger}$ Mann-Whitney $U$ test; ${ }^{+\dagger}$ Fisher's exact test; ${ }^{\ddagger} X^{2}$ test.

All patients demonstrated class II according to the Pell and Gregory classification. 


\section{Discussion}

This study evaluated the efficacy of AGS soaked in plain bupivacaine or normal saline, applied in the postextraction socket, in reducing the early postoperative pain associated with IMTM surgery. This approach is in line with Shepherd et al., who maintained that the treatment of postoperative pain following dental extraction requires a formulation that would simultaneously serve as a hemostatic agent as well as a vehicle for controlled anesthetic delivery. ${ }^{13}$ The authors tested a biodegradable dental material containing bupivacaine combined with an absorbable hemostat Gelfoam ${ }^{\circledR}$ in a rat animal model, where it was packed in post-extraction sockets, and they observed reduced post-extraction pain by assessing food consumption, body weight, and sensitivity to mechanical and thermal stimuli. ${ }^{13}$

The present study indicates that the local application of $0.5 \%$ bupivacaine hydrochloride in the manner described above provided effective pain relief in the first $4 \mathrm{~h}$ after IMTM surgery. Studies have shown that the maximum postoperative dental pain after the surgical extraction of IMTMs is usually experienced during the first 6-12 $h,{ }^{3,18}$ so the use of bupivacaine in this simpleto-apply method can be helpful for patients in the early postoperative period.

Khiavi et al. used $4 \mathrm{~mL}$ of $0.5 \%$ plain bupivacaine hydrochloride to irrigate the post-extraction sockets of IMTM patients once, before flap suturing, and compared it with normal saline irrigation in a randomized clinical study with a crossover design. ${ }^{3}$ Pain was assessed at 4 time intervals $-1,6,12$, and 24 h postoperatively. The authors reported significant pain alleviation at all time intervals, with no complications. ${ }^{3}$

In their study, Talimkhani et al. used the intra-socket administration of bupivacaine with a prepared catheter fixated to a mandibular second molar, with its cannula inserted into the socket of the extracted third molar so that the patients could directly administer drugs (bupivacaine or saline as a placebo) into the tooth socket. ${ }^{19}$ They compared it with oral mefenamic acid capsules or capsules filled with milk powder (as a placebo) in a randomized clinical study. The patients recorded the perceived pain intensity on a visual analogue scale (VAS) every $2 \mathrm{~h}$ before and after drug administration for $24 \mathrm{~h}$. The authors found that bupivacaine significantly relieved postoperative pain after the surgical extraction of IMTMs. ${ }^{19}$

To assess the clinical effectiveness of the local application of bupivacaine in relieving early post-extraction pain, the amount of improvement that is important to patients must be determined. It is not enough to consider only the statistical significance, but also whether the observed change is meaningful to patients. ${ }^{20}$ The distributionbased method was used in the present study to determine whether the pain relief achieved with the local application of bupivacaine $4 \mathrm{~h}$ after surgery was more than would be expected by chance alone, and it demonstrated that the local application of bupivacaine, as described in the present study, had a considerable clinical effect.

The difference in the pain intensity recorded $12 \mathrm{~h}$ after surgery was not statistically significant, which indicates that this method of local application of bupivacaine provided only temporary relief of postoperative pain. In the present study, postoperative pain during the first $24 \mathrm{~h}$ was assessed at 2 time intervals only ( 4 and $12 \mathrm{~h}$ after surgery) to ensure better patient compliance. Talimkhani et al. reported more prolonged pain relief at 6 time points with the use of their method of multiple intra-socket administration, which required more patient compliance, ${ }^{19}$ while Khiavi et al. reported pain relief that lasted for $24 \mathrm{~h}$ from a single irrigation of the post-extraction socket at the end of surgery. ${ }^{3}$

No major complications were encountered in this study and the difference between the 2 groups in this regard was not statistically significant, indicating that this method of local application of bupivacaine is safe to use.

\section{Limitations}

The main limitations of this study are its small sample size and the fact that the sample size estimation was not performed. However, the total number of patients in this study (40) is close to that reported in 2 other studies that investigated the local application of bupivacaine after IMTM extraction. ${ }^{3,19}$ Other limitations of the study are the fact that the operator was not blinded to the intervention and the fact that the assessment of postoperative pain during the first $24 \mathrm{~h}$ was performed at only 2 time intervals to ensure better patient compliance.

\section{Conclusions}

The local application of AGS soaked in plain bupivacaine hydrochloride significantly reduces postoperative pain $4 \mathrm{~h}$ following IMTM surgery without significant complications.

\section{ORCID iDs}

Mohanad Ali Shabat (D) https://orcid.org/0000-0001-5629-8684 Salwan Yousif Bede (1) https://orcid.org/0000-0002-0693-933X

\section{References}

1. Carter K, Worthington S. Predictors of third molar impaction: A systematic review and meta-analysis. J Dent Res. 2016;95(3):267-276. doi:10.1177/0022034515615857

2. Osunde O, Saheeb B, Bassey G. Indications and risk factors for complications of lower third molar surgery in a Nigerian teaching hospital. Ann Med Health Sci Res. 2014;4(6):938-942. doi:10.4103/2141-9248.144919

3. Khiavi RK, Pourallahverdi M, Pourallahverdi A, Khiavi SG, Oskouei SG, Mokhtari H. Pain control following impacted third molar surgery with bupivacaine irrigation of tooth socket: A prospective study. J Dent Res Dent Clin Dent Prospects. 2010;4(4):105-109. doi:10.5681/joddd.2010.027 
4. Ngeow WC, Lim D. Do corticosteroids still have a role in the management of third molar surgery? Adv Ther. 2016;33(7):1105-1139. doi:10.1007/s12325-016-0357-y

5. Kumar B, Bhate K, Dolas RS, Kumar SS, Waknis P. Comparative evaluation of immediate post-operative sequelae after surgical removal of impacted mandibular third molar with or without tube drain - split-mouth study. J Clin Diagn Res. 2016;10(12):ZC46-ZC49. doi:10.7860/JCDR/2016/20951.9054

6. Latt MM, Kiattavorncharoen S, Boonsiriseth K, Pairuchvej V, Wongsirichat $N$. The efficacy of dexamethasone injection on postoperative pain in lower third molar surgery. J Den Anesth Pain Med. 2016;16(2):95-102. doi:10.17245/jdapm.2016.16.2.95

7. Cristalli MP, La Monaca G, De Angelis C, Pranno N, Annibali S. Efficacy of preoperative administration of paracetamol-codeine on pain following impacted mandibular third molar surgery: A randomized, split-mouth, placebo-controlled, double-blind clinical trial. Pain Res Manag. 2017;2017:9246352. doi:10.1155/2017/9246352

8. Lieblich SE, Danesi H. Liposomal bupivacaine use in third molar impaction surgery: INNOVATE study. Anesth Prog. 2017;64(3):127-135. doi:10.2344/anpr-64-02-03

9. Gan TJ. Poorly controlled postoperative pain: Prevalence, consequences, and prevention. J Pain Res. 2017;10:2287-2298. doi:10.2147/JPR.S144066

10. Moore PA, Hersh EV. Local anesthetics: Pharmacology and toxicity. Dent Clin North Am. 2010;54(4):587-599. doi:10.1016/j.cden.2010.06.015

11. Dhanrajani $P$, Chung P. Comparative study of analgesia with bupivacaine $0.25 \%$ versus $0.5 \%$ for third molar removal under general anesthesia. J Dent Anesth Pain Med. 2016;16(2):117-122. doi:10.17245/jdapm.2016.16.2.117

12. Hersh EV, Moore PA. Comment on controlling dental postoperative pain and the intraoral local delivery of drugs. Curr Med Res Opin. 2015;31(12):2185-2187. doi:10.1185/03007995.2015.1109504

13. Shepherd SD, O'Buckley SC, Harrington JM, et al. A moldable sustained release bupivacaine formulation for tailored treatment of postoperative dental pain. Sci Rep. 2018;8:12172. doi:10.1038/s41598-01829696-w

14. Schulz KF, Altman DG, Moher D; CONSORT Group. CONSORT 2010 statement: Updated guidelines for reporting parallel group randomised trials. BMJ. 2010;340:c332. doi:10.1136/bmj.c332

15. Susarla SM, Dodson TB. Estimating third molar extraction difficulty: A comparison of subjective and objective factors. J Oral Maxillofac Surg. 2005;63(4):427-434. doi:10.1016/j.joms.2004.12.003

16. Yuasa $H$, Kawai $T$, Sugiura $M$. Classification of surgical difficulty in extracting impacted third molars. Br J Oral Maxillofac Surg. 2002;40(1):26-31. doi:10.1054/bjom.2001.0684

17. de Carvalho RWF, Vasconcelos BC. Pernambuco index: Predictability of the complexity of surgery for impacted lower third molars. Int JOral Maxillofac Surg. 2018;47(2):234-240. doi:10.1016/j.ijom.2017.07.013

18. Shyamala M, Ramesh C, Yuvaraj V, et al. A comparative study between bupivacaine with adrenaline and carbonated bupivacaine with adrenaline for surgical removal of impacted mandibular third molar. J Maxillofac Oral Surg. 2016;15(1):99-105. doi:10.1007/s12663-015-0791-4

19. Talimkhani I, Jamalpour MR, Babaei H, Faradmal J. Comparison of intra-socket bupivacaine administration versus oral mefenamic acid capsule for postoperative pain management following removal of impacted mandibular third molars. J Oral Maxillofac Surg. 2019;77(7):1365-1370. doi:10.1016/j.joms.2019.01.021

20. McGlothlin AE, Lewis RJ. Minimal clinically important difference: Defining what really matters to patients. JAMA. 2014;312(13):1342-1343. doi:10.1001/jama.2014.13128 